\title{
Viscoelastic Properties of Biological Materials
}

\author{
Naoki Sasaki
}

Additional information is available at the end of the chapter

http://dx.doi.org/10.5772/49979

\section{Introduction}

Almost all of biological tissues are viscoelastic and their viscoelastic mechanical properties are important in their characteristic functions. This is because constituents of tissues - cells, extracellular matrices, structural proteins, and so on - are viscoelastic. Even hard tissues have been shown to be viscoelastic. For long, main sample material for investigating the viscoelasticity has been amorphous polymeric materials, which brought about the remarkable development in phenomenological theories. Constructing mechanistic images, on the other hand, of the viscoelasticity of polymeric materials had been difficult because of dearth of the materials structural information. Contrary to these materials, for biological tissues, many structural investigations have been made and as a result detailed structural information is available. Making use of the information, it will be possible to investigate the viscoelastic properties of biological materials on the basis of their structure. Such a study will contribute to the construction of molecular theory for the viscoelasticity in amorphous materials.

In this chapter, some examples of viscoelastic nature of biological materials and then their relevance to the structure would be presented. In some cases, a mechanistic model for the viscoelasticity will be presented. As measuring method varies depending on the modulus value of the specimens, the various methods used in studying viscoelastic properties of biological materials will be illustrated.

\section{A Brief introduction to viscoelasticity}

\subsection{Introduction to elasticity and viscosity}

Elasticity is a material property that generates recovering force at an application of an external force to deform the material. When an external force is applied to a material and the material is in an equilibrium deformation, the external force is balanced by an inner force. The inner force is the recovering force. The recovering force divided by the cross sectional 
area that the external force is working on is defined as stress, $\sigma$. Suppose the material is initially in a shape of rod of length $L_{0}$ and cross-sectional area, Ao. The force is applied to the length direction and the length after the deformation is $L$. The deformation is generally normalized as,

$$
\varepsilon=\frac{L-L_{0}}{L_{0}},
$$

where $\varepsilon$ is called the engineering strain or the Caushy strain. This is used for small deformations. For small strains, a Hookean relation,

$\sigma \infty \varepsilon$

has been known to hold. The proportionality factor is defined as a modulus and the modulus is a material's constant. If the material was deformed by a tensile force, the modulus is defined as Young's modulus (Fig. 1 (a)). If the deformation was caused by a shear
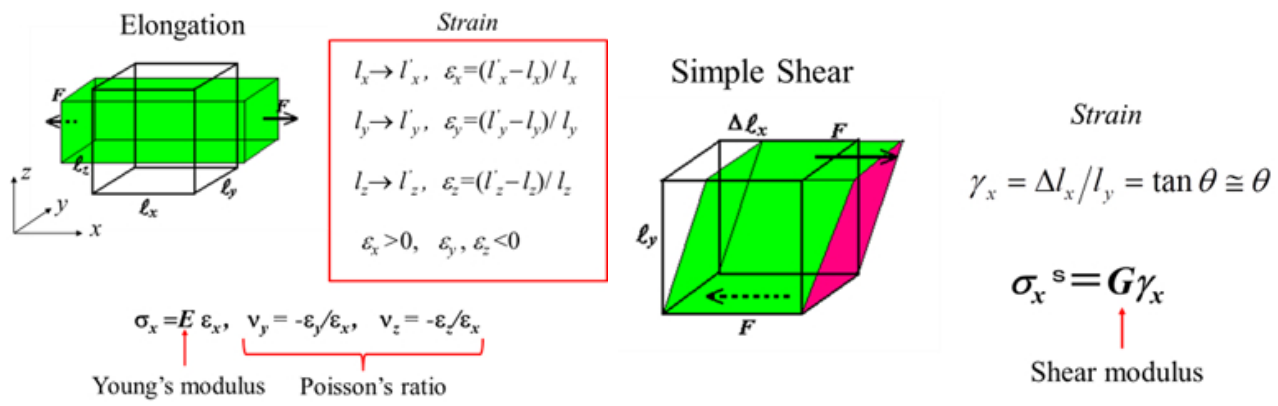

Figure 1. Deformation modes and corresponding moduli. (a) Elongation and Young's modulus, and (b) shear deformation and the shear modulus.

shear force, the modulus is the shear modulus (Fig. 1(b)). In each case, the Hookean constitutive equations are written as,

$$
\sigma=E \cdot \varepsilon \quad(E: \text { Young's modulus })
$$

and

$$
\sigma=G \cdot \gamma(G: \text { shear modulus })
$$

where $\gamma$ is the shear strain. After eliminating the external force, the recovering force and the deformation are completely diminished. Elasticity is a property of a material to resist the deformation by the external force.

Viscosity is a characteristic nature of a fluid. When a fluid is constantly flowing on an infinitively wide plain, the flow velocity is largest on the fluid surface. On the plain bottom 
the flow velocity is zero. Every part of the fluid is sheared (Fig. 2). The shear is generated by an applied force causing the flow and an internal force against the former. The internal force is also transformed into a stress generated in the flow. Newton postulated the quantitative relationship between the stress and the shear rate as,

$$
\sigma \propto \frac{d \gamma}{d t}
$$

The proportionality factor is defined as a viscosity coefficient, $\eta$, also a materials constant of a fluid. Then the constitutive relation for the viscosity is

$$
\sigma=\eta \frac{d \gamma}{d t}
$$

Viscosity is a property of a fluid to resist the force for flow. The fluid described by the equation is classified as Newtonian. After stopping the flow, the fluid maintains its deformation strain at the time of stopping. There is a saying that water conforms to the shape of the vessel that contains it.

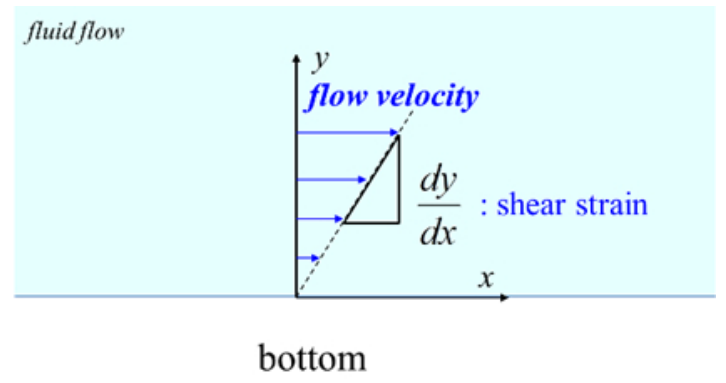

Figure 2. Shear strain in a fluid. A velocity gradient perpendicular to the bottom emerged.

\subsection{Introduction to viscoelasticity}

When a step strain excitation is applied to an idealistically elastic material, the response of the material will be that shown in Fig. 3(a). This response can be easily expected from the Hookean constitutive equation. If the same strain excitation is applied to an idealistically viscous material, the response will be those as shown in the Fig. 3(b) and (c), which is also expectable from the Newtonian constitutive equation. Materials that exhibit viscoelastic properties can be considered as having both elastic and viscous components. Then, in the case of viscoelastic materials, the response is expected to have both characteristics of elasticity and viscosity. Schematic drawing of the stress response according to many experimental results is shown in Fig. 4. Here we define the stress relaxation as follows. Fig. 5 shows a step strain excitation and a stress response to it for a viscoelastic material. The instantaneous and equilibrium moduli, $m_{\mathrm{g}}$ and $m_{\mathrm{e}}$, respectively, are material's constants. The relaxation function $\phi(t)$ is a material's function. Phenomenological investigation of materials from the stand point of viscoelastic properties is to determine these material's constants and 
function and, then, deduce the molecular mechanism corresponding to the viscoelastic behavior. For this purpose, more generalized tool shall be introduced. Any given strain excitation can be constructed by a linear combination of many small step strains applied to the material at every $\Delta \mathrm{t}$ seconds. According to the linear response theory, stress response to the excitation also can be a linear combination of responses to small step strains applied to the material (Fig. 6). From any given strain excitation $\varepsilon(t)$, stress response $\sigma(t)$ would be obtained through the Boltzmann's superposition equation,

$$
\sigma(t)=\int_{-\infty}^{t} \frac{d \varepsilon(u)}{d u} m(t-u) d u .
$$

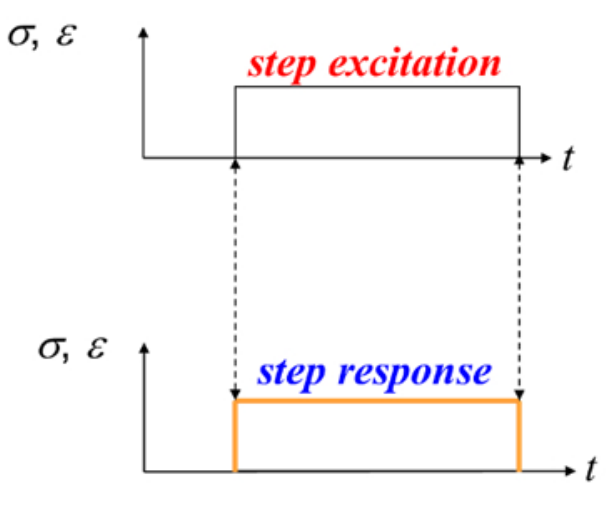

(a)

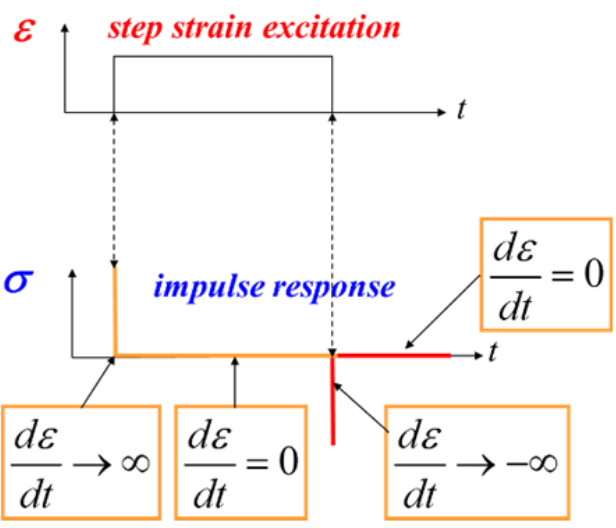

(b)

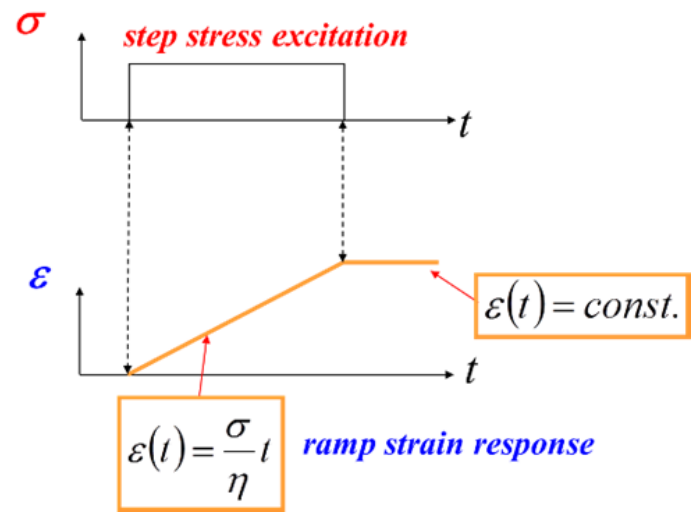

(c)

Figure 3. Responses of idealistically elastic materials (a) and that of idealistic viscous materials (b) and (c) to step strain and stress excitations, respectively. 


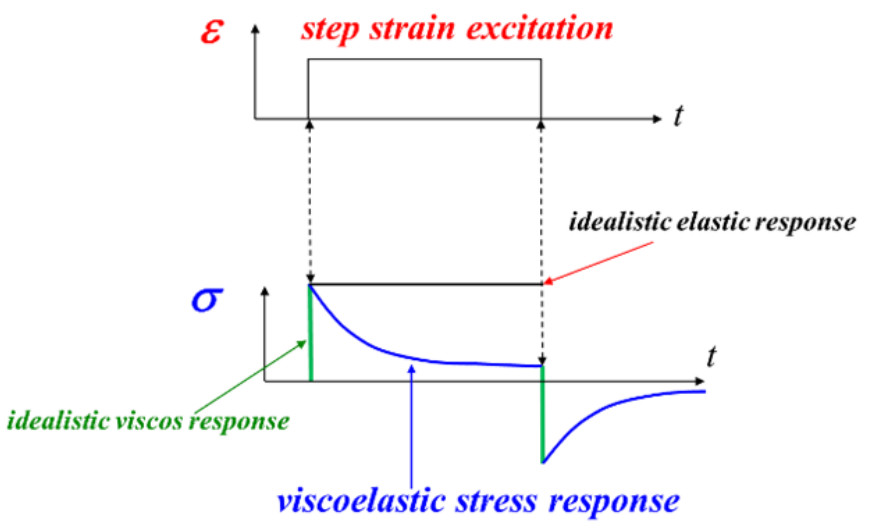

Figure 4. Response of viscoelastic materials to a box-shaped strain excitation.

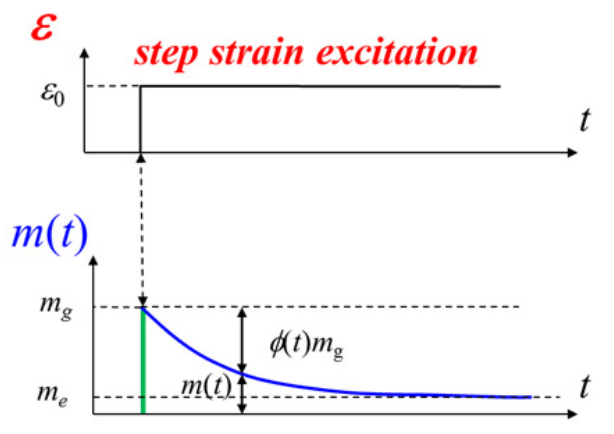

$$
\begin{aligned}
& \text { relaxation modulus } \\
& m(t)=\frac{\sigma(t)}{\varepsilon_{0}}=m_{g}[1-\phi(t)] \\
& \phi(t): \text { relaxation function } \\
& m(0)=m_{\mathrm{g}} \quad \text { : glass modulus } \\
& m(\infty)=m_{\mathrm{e}} \quad: \text { equilibrium modulus }
\end{aligned}
$$

Figure 5. Definition of the response of a viscoelastic material to a step strain excitation.

\section{Strain excitation}

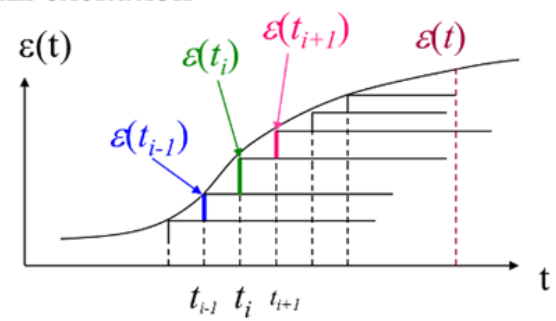

\section{Stress response}

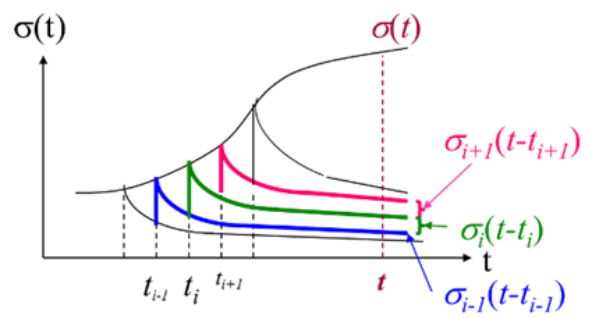

Figure 6. Generalized strain excitation and the stress response of viscoelastic materials to the former. From the relationship between the excitation and the response, Boltzmann's equation, eq. 7, was derived. 
For stress relaxation experiments, a step strain excitation is needed. But, actually, it is difficult to realize such an excitation strain. The realistic one should be a lamp followed by a plateau strain. An acceptable lamp width can be determined from the Boltzmann equation above. If the relaxation function is described as

$$
\phi(t)=1-\exp (-t / \tau)
$$

where $\tau$ is a characteristic relaxation time of a material, the stress response will be

$$
\sigma(t)=A \tau e^{-t / \tau}\left(e^{t_{1} / \tau}-1\right)
$$

In this equation, $A$ is a constant and $t_{1}$ is the lamp width. If $t_{1} \ll \tau$, it is rewritten as

$$
\sigma(t)=A t_{1} \exp (-t / \tau)
$$

which is a usual relaxation modulus form of materials with single relaxation time $\tau$ as a response to the step strain excitation.

\subsection{Viscoelastic investigation}

As indicated above, the objective of the viscoelastic studies of materials is to understand the molecular mechanism corresponding to the viscoelastic behavior. For this purpose, to know the relaxation function of the material is needed. Almost all actual relaxation data, however, cannot be described by a relaxation function with single relaxation time as shown by eq. 8 . In order to describe the relaxation modulus, then the relaxation function, the following methods have been traditionally employed: (A) multi relaxation time analysis, (B) relaxation time distribution analysis (Ferry, 1980), and (C) analysis using specific functions.

(A) and (B) are substantially similar analyses. For the analysis (A), experimental relaxation modulus, $E(t)$, is fitted by

$$
E(t)=E_{e}+\sum_{i=1}^{N} a_{i} \exp \left(-t / \tau_{i}\right)
$$

where $E_{\mathrm{e}}$ is an equilibrium modulus. If $N$ is large and $a_{i}$ is a continual function of $\tau, a(\tau)$, eq. 11 can be described as

$$
E(t)=E_{e}+\int_{-\infty}^{t} a(\tau) \exp (-t / \tau) d \tau
$$

This description represents the relaxation time distribution analysis and $a(t)$ is a relaxation time spectrum. By a numerical transformation of the experimental data, the relaxation spectrum will be obtained. At this point, relaxation spectrum does not have any more information than the original relaxation data. It is still difficult to deduce molecular events underlying the relaxation. 
For (C), it has been established that the non-single relaxation timed relaxation process can be well described by a power law relation or stretched exponential function, depending on the materials and their state;

$$
E(t)=E_{0} t^{-n}+E_{e} \quad(0<n \leq 1)
$$

or

$$
E(t)=E_{0} \exp \left[-(t / \tau)^{\beta}\right](0<b \leq 1) .
$$

One of the advantages of using these functions for describing the relaxation process is easily to establish the mechanistic model for the relaxation process. As these two functions have been found to describe well the mechanical or electric relaxation phenomena in many amorphous glassy materials, they are regarded as universal functions.

When there is a dynamic structural inhomogeneity in glassy materials, Jonscher proposed that the power in the power law relation, eq. 13, indicates the correlation of molecular motions among motional groups and the variation in the number of motional unit in each molecular motional group (Jonscher, 1983). On the other hand, for explanation of the exponent value in the stretched exponential function, eq. 14, a diffusion-trap model is proposed (Klafter et al., 1986; Philips, 1996). When a step strain is applied to a material, many excited sites will emerge inside the material. In amorphous glassy materials, molecular motion of constituents could be regarded as a diffusion of a free volume of the constituents. When the free volume comes to an excited site, the site will be relaxed. This would be an elementary process of the relaxation. If the excited sites in the material is distributing on a fractal lattice, total relaxation of the material is expected to be described by the stretched exponential function, eq. 14. The exponent value, $\beta$, is strongly related the geometry of the exciting site distribution (Potuzak et al., 2011).

\section{Stress relaxation experiments}

In the introductory section, only the stress response of materials to strain excitation was explained. This is because our viscoelastic study of biological materials mainly has been employing the stress relaxation experiments. Therefore, in this chapter, the relaxation modulus of biological materials is discussed in conjunction with their structural information. In this section, the empirical determination of the relaxation modulus of materials would be explained.

\subsection{Apparatus and equipments}

Figure 7 shows a schema for the stress relaxation experiments. By moving the load cell (LC), Kyowa Electric Works LTS series, strain is applied to the specimen through an equipped attachment mounted on a probe and the stress response to the excitation is detected by LC. LC is mounted on an auto micro-stage, Sigma Koki CTS-50X, derived by a stage controller Sigma Koki MARK-12. The sensitivity of LC and the attachments are chosen depending on 
the specimens. Soft materials are examined by a tension or indentation methods depending on the specimen. When the specimen can be shaped in a sheet, relaxation modulus was measured by the tensile strain application.

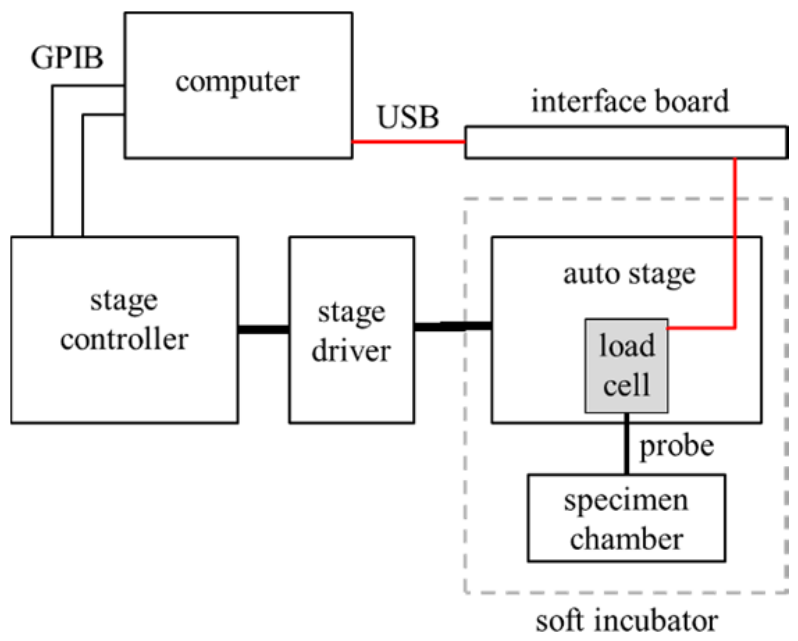

Figure 7. Block diagram for stress relaxation measuring apparatus.

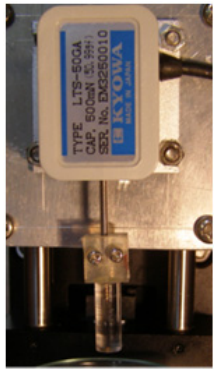

(a)

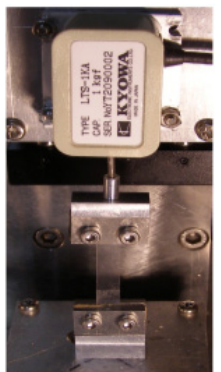

(b)

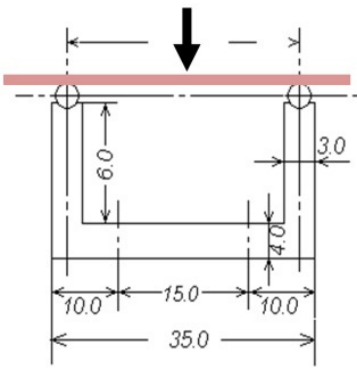

(c)

Figure 8. (a) shows the attachments for tension measurements. Cell-seeded gels are examined by the indentation. Fig. 8(b) shows the indenter for this measurement. Hard materials are shaped in bean examined by a bending method. Fig. 8(c) shows three point bending equipment used for stress relaxation of bone specimens. In both measurements, specimens are soaked in Ringer's solution at $37^{\circ} \mathrm{C}$ during the relaxation experiments. Detected LC signals were passed to adata logger, Kyowa Electric Works PCD-300B, and finally stored into a PC.

\subsection{Measurements}

At each measuring mode, prior to the start of the stress relaxation experiment, an upper clump in the case of tensile strain application and an indenter head in the cases of indentation and beam bending must be placed at a zero strain point. For this procedure, the 
clump or indenter head were derived in $5 \mu \mathrm{m}$ step. The judgment for an initial touch of the probe head on the specimen was made on the indication of signals from LC starting to change to more than a few $\mu \varepsilon$. The stress relaxation experiments were started one hour after the zero strain state detection, waiting for stress relaxations brought about by the zero point detecting procedures. For the stress relaxation measurement, strain values less than $1 / 2$ of yield strain value were applied to the specimen at the deformation rate of $3.3 \mathrm{~mm} \mathrm{sec}^{-1}$.

\section{Viscoelastic properties of agarose gel and cell-seeded agarose gel}

Agarose gel is a material of multi-purpose use. For example, it has been used widely as a cell culture matrix. In the following sections, how cultured cells in agarose gel change the mechanical properties of matrix agarose gel by precipitating extracellular matrix would be shown. For the precise estimation of the change in mechanical properties of the matrix, those of agarose gel itself must be quantitatively estimated. Here results obtained for pure agarose gel at first and then cell-seeded ones would be presented. Agarose used in this experiment and those in the following sections is type VII agarose for cell culture use purchased from Sigma.

\subsection{Agarose gel}

Recent investigation on the agarose gelation revealed that a phase separation takes place slightly below the gelation temperature, where the gelation process was expected to proceed competitively with the phase separation of the solution (Morita et al., 2008). It is expected that the mechanical properties of so prepared agarose gel depend remarkably on the thermal history during the preparation. For the reproducible data, gel preparation must be carefully conducted. Agarose type VII powder (Sigma Co. Ltd) was dissolved in distilled and deionized water and stirred for $12 \mathrm{hrs}$ at room temperature. The aqueous solution was then incubated at $90^{\circ} \mathrm{C}$ for $5 \mathrm{hrs}$. The incubated solution was quenched from $90^{\circ} \mathrm{C}$ to $4{ }^{\circ} \mathrm{C}$, where the gelation temperature was determined to be $32^{\circ} \mathrm{C}$. Before mechanical test, the gel specimens were swollen with the distilled and deionized water. Quench temperature $4^{\circ} \mathrm{C}$ is far below the spinodal point curve in the phase diagram. Fig. 9 shows the relaxation modulus of $2 \%(\mathrm{w} / \mathrm{v})$ of Type VII agarose gel plotted against time. The modulus value was estimated by using the Hayes equation,

$$
E=\frac{F\left(1-v^{2}\right)}{2 \text { dak }},
$$

where $F$ is the recovering force of gel applied to the indenter surface, $a$ is the radius of the indenter and $d$ is the deformation depth (Hayes et al., 1972). For a specimen of thickness $h, \kappa$ is a function of the ratio, $a / h$, and Poisson's ratio, $v$, of the gel. Following Watase et al., Poisson's ratio of our agarose gel was considered to be very close to 0.5 because of its near incompressibility (Watase et al., 1983). $\kappa$ comes from the solution of the integral equation, and its value is numerically calculated and tabulated (Hayes et al., 1972). The radius of the 
indenter head used in this work was $a=3 \mathrm{~mm}$, and the average thickness of specimens was $h=3 \mathrm{~mm}$. Since Poisson's ratio was assumed to be $0.5, \kappa$ of 3.609 was employed. There is a large relaxation up to $10^{4} \mathrm{sec}$ followed by a plateau which is characteristic of gels. As both axes are scaled in logarithm, it is clear that the relaxation modulus is not described by a power law relation. After examining the data, the empirical equation

$$
E(t)=E_{0} \exp \left[-(t / \tau)^{\beta}\right]+E_{e} \quad(0<\leq 1)
$$

has been found suitable. In the Fig. 9, determined parameter values are listed. Recent investigation on the stretched exponential function revealed that $\beta$ value suggests the relaxation mechanism at molecular level for homogenous systems: $\beta=3 / 5$ for relaxation arising from short-range forces and $\beta=3 / 7$ for relaxation dominated by long-range forces (Philips, 1996). According to $\beta \sim 0.4$ obtained here for agarose gel, the driving force for the relaxation would be long-range ones.

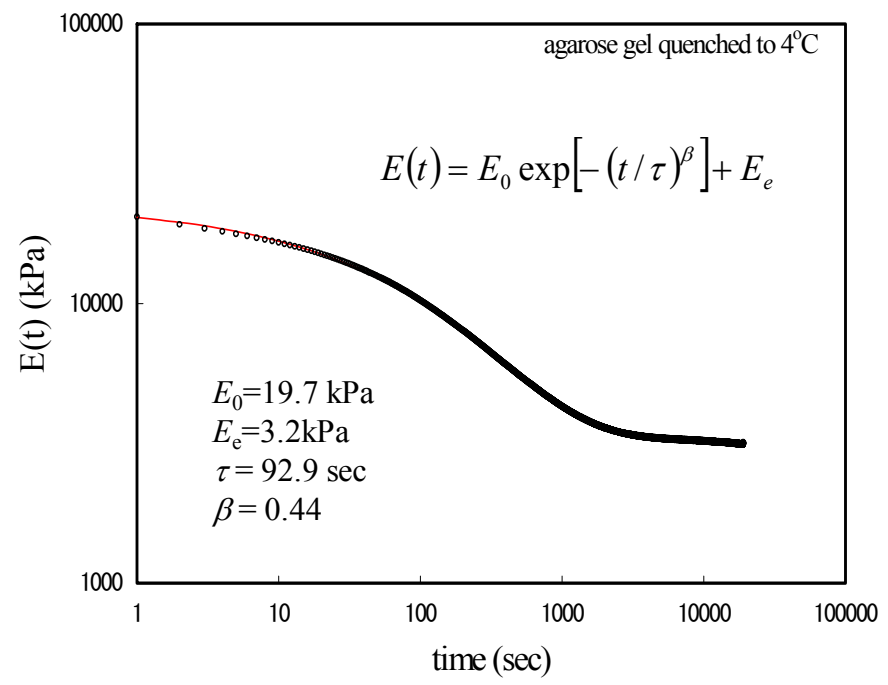

Figure 9. Relaxation modulus curve for agarose gel fabricated by quenching to $4^{\circ} \mathrm{C}$.

\subsection{Osteoblast seeded agarose gel}

On the basis of the relaxation of agarose gel indicated above, how cells change their matrix by precipitating organic and inorganic materials has been investigated (Hanazaki et al., 2011). As seeded cells were osteoblast-like cells, MC3T3-E1, the system can be regarded as a model system of osteogenesis. MC3T3-E1 cells precipitate collagenous proteins and hydroxyl apatite (HAp)-like minerals. Fig.10 shows stress relaxation curves for osteoblasts seeded agarose gel cultured up to 18 days. Relaxation curve for agarose gel is also plotted as 
a control. All specimens showed the characteristic stress relaxation curve of agarose gel; a large relaxation up to $10^{4} \mathrm{sec}$ followed by a gel plateau where the former was attributed to molecular motions of polymer chains between two adjacent cross-links of the gel and the latter to the elasticity of the gel network. Each relaxation modulus curve was able to be described well by eq.16, and parameter determined by the fitting was listed in Table 1 . Change in modulus value both $E_{0}$ and $E_{\mathrm{e}}$ as a function of culturing period was not so simple. Up to day 15, the change was not significant and at day 18, both modulus values significantly increased. The relaxation time $\tau$ and its distribution $\beta$ did not change significantly through the cultivation period. Fig. 11 shows X-ray diffraction profiles of the cell-agarose gel composite specimens cultured for the indicated periods. The profile for a sintered HAp is also shown as a reference (Okazaki et al., 1997). There is a large peak at about $2 \theta \sim 20^{\circ}$, which was attributed to the diffraction peaks related to the agarose gel matrix (Foord \& Atkins, 1989). The diffraction profile of the specimen after 9 days of culture showed diffraction peaks attributable to HAp crystal, where the peak at 20 25 was indexed as (002) and those at $32^{\circ}$ were indexed as (211), (112), and (300) (Okazaki et al., 1997). With further extension of culturing time, the intensities of peaks corresponding to HAp crystal gradually increased. In Figs. 12(a) and 12(b), Eo and Ee values are respectively plotted against culture days. Relative integrated diffraction intensity in Fig. 11 is also plotted against days, defined as the ratio of intensity from 002 plan at each culture day against that at the day $18, I_{002} / I_{002}(18)$. The diffraction intensity is generally proportional to the amount of crystals, provided that the state of crystals in each specimen is not greatly different (Kakudo \& Kasai, 1972). In Fig. 11, the half width of each (002) peak was almost the same throughout the culture time up to 18 days. In this case, the intensity can be considered to be proportional to the amount of HAp crystals in the specimen. The I002/I002(18) vs. culture time plot indicates that the amount of HAp crystals increases monotonically with culture period. The result indicates that even though HAp content proportionally increases with culture time, modulus values increment started only from the day 18. To explain this discrepancy between HAp increment and modulus values increment, the efficiency of the reinforcement of agarose gel matrix by precipitated mineral particles was appreciated. Fig. 13 shows a schema of the precipitation process of mineral particles into agarose gel matrix. In the early stage of HAp particle precipitation by MC3T3-E1 cells, the number of mineral particles increases inside a gel network [schema (a) in Fig. 13]. In this state of a cell-seeded agarose gel system, precipitated mineral particles do not contribute to the composite modulus. When a mineral particle cluster percolates inside the gel network, the modulus of the composite will increase [schema (b) in Fig. 13]. The percolation could occur almost simultaneously in every network around each cell. The effect of this percolation is considered to be similar to the increase in the crosslink density, which will lead to the increase in $E_{e}$. At the same time, the stiffness of network chains around the mineral percolation would be increased and their mobility would be decreased by the precipitated mineral particles. The empirical equation employed in this study, eq. 16, basically assumes that a relaxing entity can be described by a serial combination of a spring and a dashpot, where the former represents an elasticity, $\varepsilon$, and the latter a viscosity, $\eta$, in a material. The relaxation time, $\tau$, is expressed as 


$$
\tau=\frac{\eta}{\varepsilon}
$$

An increase in the stiffness of the network chain implies an increase in $\varepsilon$ and a decrease in their mobility, that is, an increase in $\eta$, which would result in an increased $E_{0}$ with relaxation time almost unchanged. Above the percolation threshold, the increase in the modulus of the system, (both $E_{0}$ and $E_{e}$ values) with culture time would become remarkable. In our case, the percolation was considered to take place between days 15 and 18 of culture.

\begin{tabular}{cccccc}
\hline $\begin{array}{c}\text { culture } \\
\text { period (day) }\end{array}$ & sample size & $E_{0}(\mathrm{kPa})$ & $\tau(\mathrm{sec})$ & $\beta$ & $E_{e}(\mathrm{kPa})$ \\
\hline agarose & 4 & $16.7 \pm 0.6$ & $162 \pm 17$ & $0.49 \pm 0.01$ & $2.19 \pm 0.32$ \\
1 & 4 & $15.1 \pm 2.4$ & $178 \pm 61$ & $0.43 \pm 0.03$ & $2.36 \pm 0.38$ \\
9 & 4 & $13.9 \pm 3.0$ & $144 \pm 54$ & $0.44 \pm 0.04$ & $2.56 \pm 0.40$ \\
12 & 4 & $12.4 \pm 2.0$ & $168 \pm 59$ & $0.48 \pm 0.03$ & $2.21 \pm 0.41$ \\
15 & 4 & $15.0 \pm 2.0$ & $136 \pm 27$ & $0.46 \pm 0.01$ & $2.22 \pm 0.13$ \\
18 & 4 & $21.3 \pm 3.6$ & $173 \pm 41$ & $0.49 \pm 0.02$ & $3.38 \pm 0.16$ \\
\hline
\end{tabular}

(From Hanazaki et al., J. Biorheology. In press. DOI 10.1007/s12573-011-0043-2. With permission.)

*All the parameter values are presented as the average value \pm the standard error.

Table 1. Parameter values determined by fitting equation (16) to experimental data.

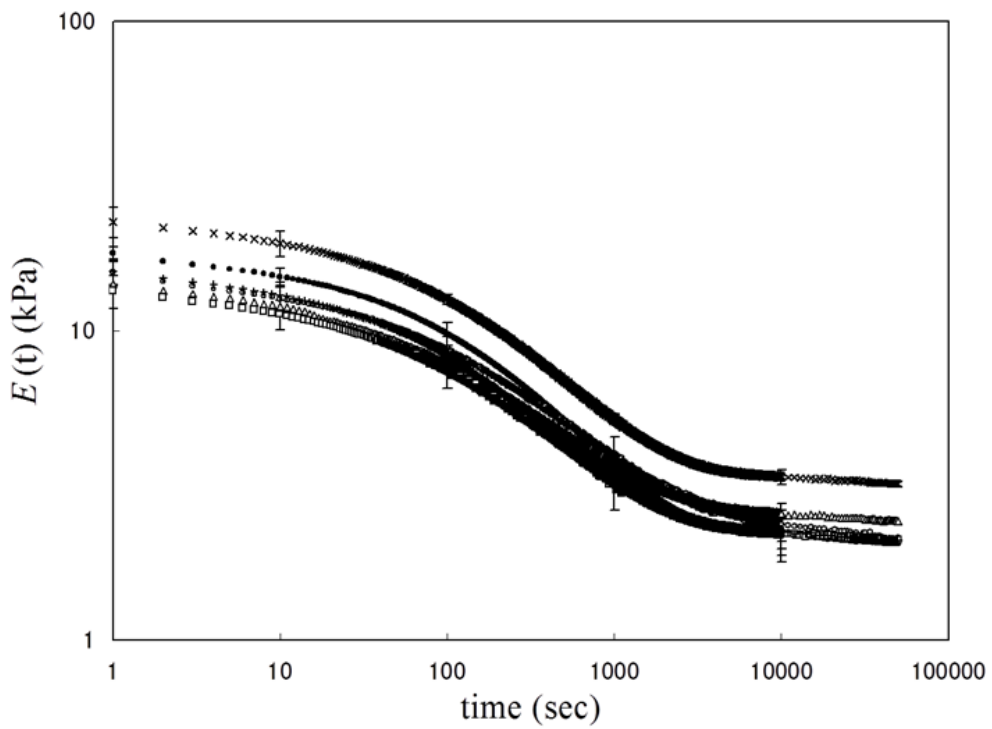

Figure 10. Relaxation modulus curves for MC3T3-E1 seeded agarose gels cultured for 1 day ( $\circ$ ), 9 days $(\Delta), 12$ days $(\square), 15$ days $(+)$, and 18 days $(x)$. Relaxation modulus for agarose gel $(\bullet)$ is also shown as a control. (From Hanazaki et al., J. Biorheology. In press. DOI 10.1007/s 12573 -011- 0043-2. With permission.) 


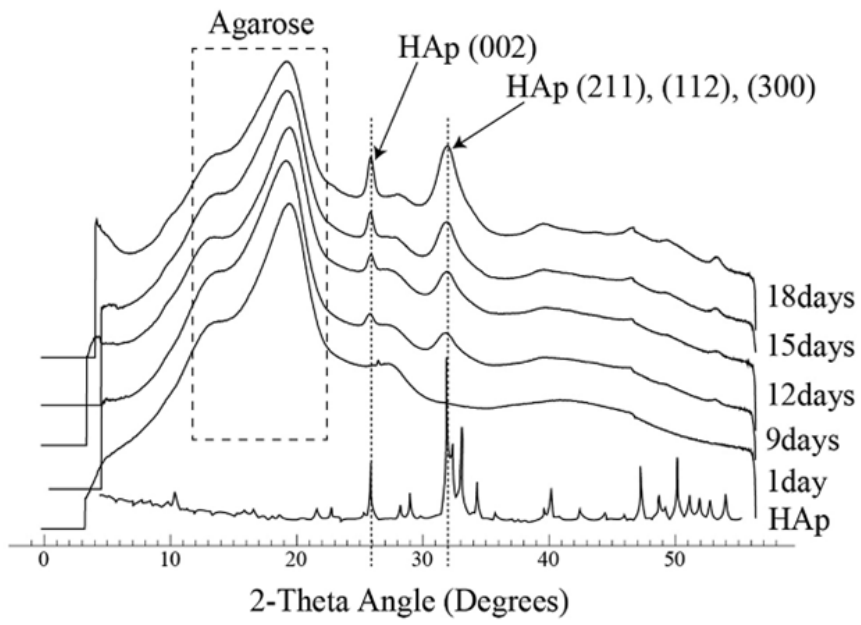

Figure 11. X-ray diffraction profiles for cell-seeded agarose gel cultured for indicated days. That for HAp powder is also shown for reference (Okasaki at al., 1997). (From Hanazaki et al., J. Biorheology. In press. DOI 10.1007/s12573 -011- 0043-2. With permission.)
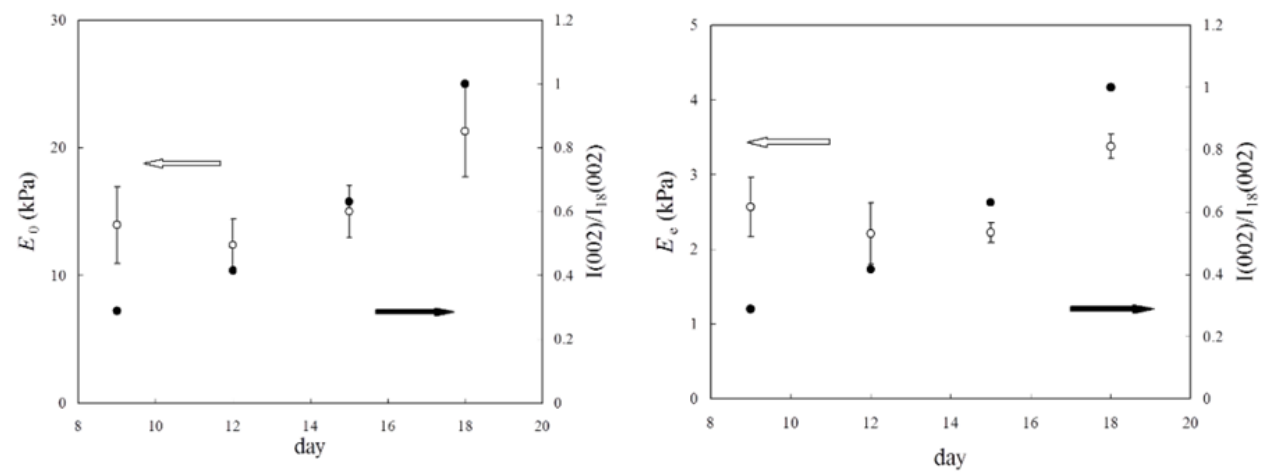

Figure 12. Comparison of $E_{0}(\mathrm{a})$ and $E_{\mathrm{e}}(\mathrm{b})$ values with diffraction intensity for HAp 002 as a function of culture period. (From Hanazaki et al., J. Biorheology. In press. DOI 10.1007/s12573-011-0043-2. With permission.) 
(a)

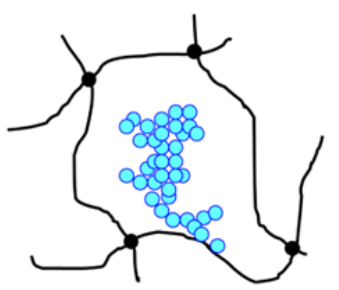

(b)

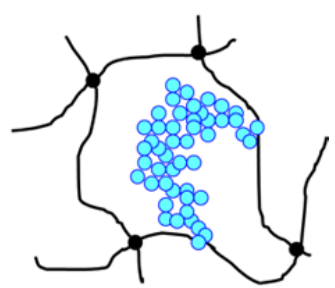

- HAp particle

gel network chain

Figure 13. A model explaining the discrepancy of the mechanical behavior from the $\mathrm{X}$-ray diffraction profiles as a function of culture period. (a) Precipitation of HAp in agarose gel up to day 15. (b) Partial percolation of HAp precipitates in a agarose net work area after day 18. (From Hanazaki et al., J. Biorheology. In press. DOI 10.1007/s12573-011-0043-2. With permission.)

\subsection{Chondrocyte seeded agarose gel}

Chondrocytes are responsible for the synthesis, maintenance, and gradual turnover of an extracellular matrix (ECM) composed principally of a hydrated collagen fibril network enmeshed in a gel of highly charged proteoglycan molecules. Each chondrocyte is surrounded by a narrow tissue region of pericellular matrix (PCM), the elastic modulus of which has been regarded to be larger than that of a chondrocyte and smaller than those of territorial matrix (TM) and inter territorial matrix (ITM). The unit consisting of a chondrocyte with PCM is generally termed a chondron. Since the volume fraction of chondrocytes in articular cartilage has been reported to be about $5-10 \%$, chondrons will occupy more than this volume fraction. Even though the modulus of a chondron is much smaller than those of TM and ITM, the contribution of chondrons as mechanical elements to the mechanical function of articular cartilage is not negligible. To clarify the contribution of the viscoelastic nature of chondrons to that of articular cartilage tissue, relaxation modulus for chondrocyte-seeded agarose gel and that cultured for three weeks were measured as a model system of articular cartilage (Sasaki et al., 2009). The relaxation modulus curves for chondrocyte-seeded agarose gel were compared with that for agarose gel (AG). Fig. 14(a) shows a microscopic image of chondrocytes-seeded in agarose gel (AGC0) that was obtained immediately before mechanical measurements. The density of chondrocytes was $30 \times 10^{6} \mathrm{cell} / \mathrm{ml}$ and the average size of a chondrocyte was about $10 \mu \mathrm{m}$ in diameter. Chondrocytes were shown to be dispersed almost homogeneously. Fig. 14(b) shows a microscopic image of chondrocyte-seeded agarose gel after 21 days of cultivation (AGC3). The magnification is the same as that in Fig. 14(a). Toluidine Blue staining was performed. Material was synthesized around each cell after 21 days of cultivation. Fig. 15 shows the relaxation modulus of AGC3 compared with those of AGC0 and AG. The relaxation modulus of AGC0 was increased by cultivation to be that of AGC3. In a short time region, up to $10^{2} \mathrm{sec}$, the increment was not statistically significant. After $10^{3} \mathrm{sec}$, the relaxation 
modulus value of AGC0 significantly increased $\left({ }^{*} \mathrm{p}<0.05\right)$. In order to analyse the change in the viscoelastic properties of chondrocyte-agarose composite originated from the PCM-like material production by chondrocytes, the differences in the relaxation modulus values among three samples were calculated. Fig. 16 shows the difference in the relaxation modulus values between AG and AGC0 (०),

$$
\Delta E_{\mathrm{AGC} 0-\mathrm{AG}}=E_{\mathrm{AGC} 0}-E_{\mathrm{AG}}
$$

\section{AGC3 and AGC0 ( $\mathbf{\Delta})$,}

$$
\Delta E_{\mathrm{AGC} 3-\mathrm{AGC} 0}=E_{\mathrm{AGC} 3}-E_{\mathrm{AGC} 0}
$$

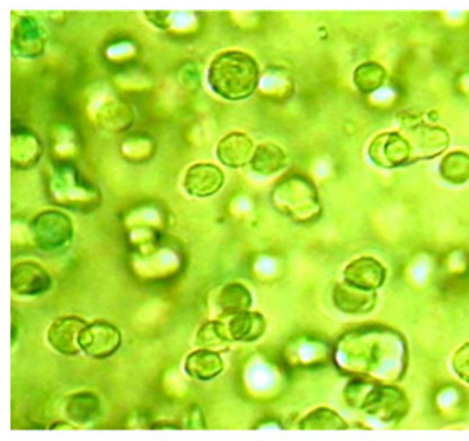

$\overline{10 \mu \mathrm{m}}$

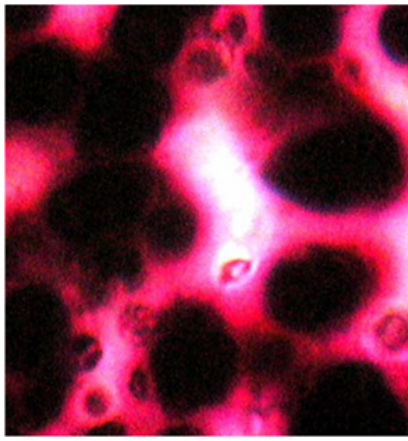

$$
\overline{10 \mu \mathrm{m}}
$$

Figure 14. Microscopic images of chondrocyte-seeded agarose gel (a) before culture and (b) cultured for 21 days.(From Sasaki et al., J. Biorheology 23, 95-101 (2009). With permission.)

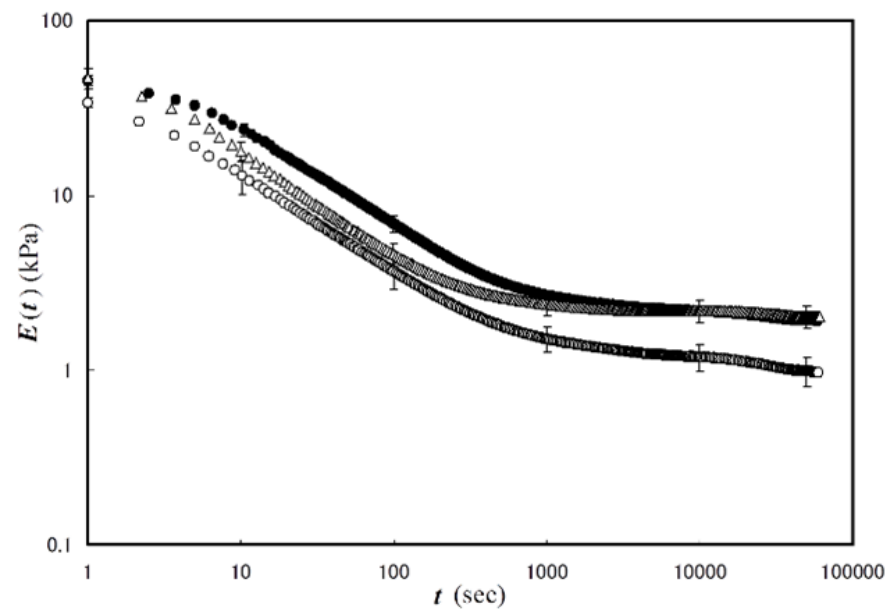

Figure 15. Relaxation modulus curves for chondrocyte seeded agarose gel immediately after seeded $(\circ)$ and that cultured for 3 weeks $(\Delta)$. Relaxation modulus curve for agarose gel is also shown as a reference (•). (From Sasaki et al., J. Biorheology 23, 95-101 (2009). With permission.) 


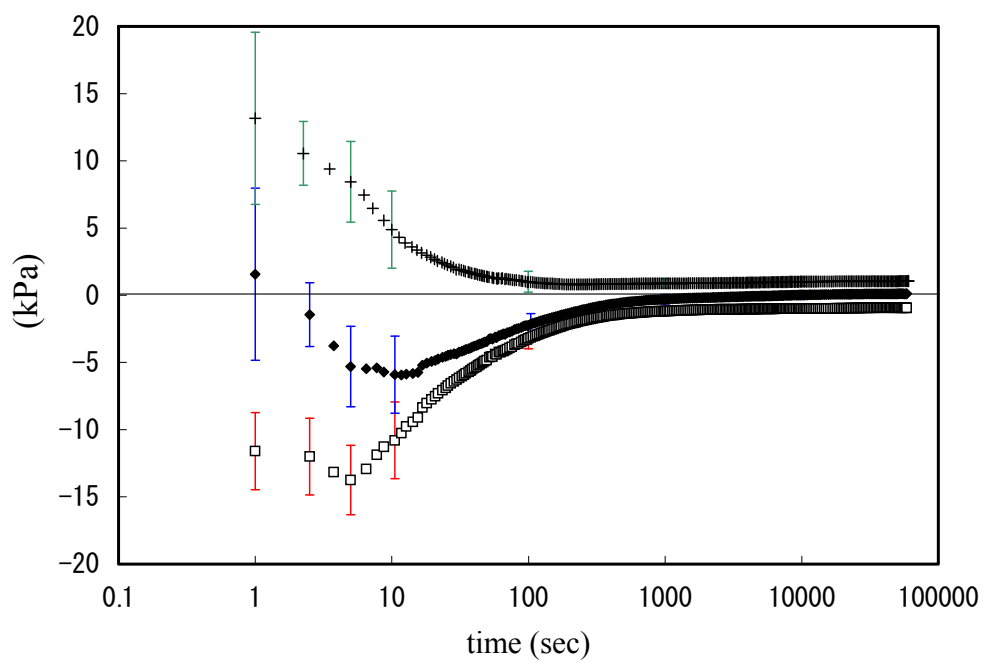

Figure 16. Differences of relaxation modulus curves. $\operatorname{EAGCO}_{\mathrm{A}}(\mathrm{t})-\mathrm{E}_{\mathrm{AG}}(\mathrm{t})(\mathrm{o}), \mathrm{E}_{\mathrm{AGC}}(\mathrm{t})-\mathrm{E}_{\mathrm{AG}}(\mathrm{t})(\bullet)$, and $\mathrm{EAGC3}_{(\mathrm{t})}-\mathrm{EAGCo}_{(\mathrm{t})}(\dagger)$. (From Sasaki et al., J. Biorheology 23, 95-101 (2009). With permission.)

and AGC3 and AG $(\diamond)$,

$$
\Delta E_{\mathrm{AGC} 3-\mathrm{AG}}=E_{\mathrm{AGC3}}-E_{\mathrm{AG}}
$$

where subscript letters AG, AGC0, and AGC3, indicate the relaxation modulus observed for AG, AGC0, and AGC3 specimens, respectively. The difference was remarkable in the gel viscoelasticity region. In $\Delta E_{\mathrm{AGCO}-\mathrm{AG}}$, as the modulus of $\mathrm{AG}$ is larger than that of AGC0, the difference was negative, showing the reduction of relaxation modulus by the seeding of chondrocytes. In the Figure, the modulus value difference looks to become levelled off in the short time region from $1 \mathrm{sec}$ to more than $10 \mathrm{sec}$. The characteristic relaxation time of agarose gel has been reported to be around $10^{2} \mathrm{sec}$ (Watase et al., 1980), while that of a chondrocyte was determined to be $1 \sim 30 \mathrm{sec}$ (Leipzig et al., 2005; Jones et al., 1999; Shieh et al., 2006). The behaviour of $\Delta E_{A G C 0-A G}$ in the short time region may be a reflection of the viscoelastic chondrocyte contribution as an elastic component in short time region of the relaxation process. $\Delta E_{\mathrm{AGC}-\mathrm{AGC}}$ represents the modulus increase by the PCM-like material production by chondrocytes. The modulus generally increased throughout the relaxation process. However, the increment also seems to be levelled off in the short time region as the reduction in $\Delta E_{\mathrm{AGCO}-\mathrm{AG} .} \Delta E_{\mathrm{AGC} \text {-AG }}(\diamond)$ shows the re-increase in the relaxation modulus from AGC0 by the production of PCM-like material by chondrocytes. The plateau modulus value of AGC3 at times larger than $10^{3} \mathrm{sec}$ almost completely recovered the value of AG (the horizontal axis of $\Delta E=0$ ), indicating that the gel modulus value once reduced by the chondrocyte seeding was increased with the PCM-like material production by chondrocytes after 21 days of cultivation. On the other hand, as $\Delta E_{\mathrm{AGC3}-\mathrm{AG}}=\Delta E_{\mathrm{AGCO}-\mathrm{AG}}+\Delta E_{\mathrm{AGC3}-\mathrm{AGC}}$, the increment from $\Delta E_{\mathrm{AGC}-\mathrm{AG}}$ was also shown to be significant in both the plateau ( $t$ larger than $\left.10^{3} \mathrm{sec}\right)$ and the relaxation region (less than $\left.10 \mathrm{sec}\right)\left({ }^{*} \mathrm{p}<0.05\right.$ and ${ }^{* *} \mathrm{p}<0.01$, respectively). The 
change in the relaxation modulus in $\Delta E_{\mathrm{AGC} 3-\mathrm{AGC}}$ can be attributed to the material produced by chondrocytes after 21 days of cultivation. For the increase in gel modulus value in general, two possibilities would be responsible; (1) the increase in the modulus of gel network conforming polymer chain and (2) the increase in the cross-link density. The case (1) would result in a shortened relaxation time and the case (2) would provide a larger equilibrium modulus. In AGC3, the specimen seems to obtain a larger value of equilibrium modulus by the production of PCM-like material. According to Bushmann et al. (1995), the PCM-like material produced by chondrocytes was firstly deposited in the intra-network space of agarose gel in the vicinity of each chondrocyte surface in early cultures and with the cultivation period the material deposition area extended. From the stress relaxation results above, it is deduced that the PCM-like material in AGC3 could provide apparent cross-link points, which would contribute to the increase in the apparent cross-link density as compared with that in AGC0.

\section{Stress relaxation of bones}

In the previous section, viscoelastic properties of model systems of cartilaginous and osteogenesis tissues were shown. In this section, viscoelastic mechanical properties of actual tissues, in particular bones, would be described.

\subsection{Relaxation modulus of cortical bone}

Bone has been often regarded mechanically as a composite material of hydrated organic matrix mainly composed of collagen and hydroxyapatite (HAp)-like mineral phase. It is thought that the pliant collagen is reinforced by stiff mineral particles, and, as a composite, the brittleness of the mineral is compensated for by the viscoelasticity of the collagen. Recently, the existence of non-collagenous glue proteins that connect mineralized collagen fibres has been revealed (Fantner et al., 2005). These organic phases have been suggested to be responsible for the toughness of bone. Because of this viscoelasticity of collagen fibres and non-fibrous proteins in the bone matrix, bone itself has noticeable viscoelasticity (Currey, 1965; Sasaki, 2000). Detailed experimental studies on the viscoelasticity of bone have been carried out only recently, despite the fact that it has been known for a long time that bone is viscoelastic (Currey, 1964; Lakes et al., 1979). According to the results of those experimental studies, the relaxation modulus of bone could not be adequately described by a single relaxation time and was found, unlike synthetic polymeric materials, to be thermorheologically complex (Vincent, 1982).

In our previous papers, as a new empirical equation for the description of stress relaxation of cortical bone, we proposed that stress relaxation of cortical bone could generally be described by a linear combination of two Kohlraush-Williams-Watts (KWW) functions (Iyo et al., 2004; Iyo et al., 2006),

$$
E(t)=E_{0}\left\{A \exp \left[-\left(t / \tau_{1}\right)^{\beta}\right]+(1-A) \exp \left[-\left(t / \tau_{2}\right)^{\gamma}\right]\right\},[0<A, \beta, \gamma<1],
$$


where $E_{0}$ is the initial modulus value, $E(0) . \tau_{1}$ and $\tau_{2}\left(>>\tau_{1}\right)$ are characteristic times of the relaxation processes, $A$ is the fractional contribution of the fast relaxation to the whole relaxation process, and $\beta$ and $\gamma$ are parameters describing the shape of the relaxation modulus. It has been revealed that the first term represents the relaxation in the collagen matrix in bone and the second term is related to the change in a higher-order structure of bone that is responsible for the anisotropic mechanical properties (Iyo et al., 2004). It seems to be possible to relate the viscoelastic properties and the hierarchical structure of bone by investigating these mechanical parameters. In this section, the effect of the structural anisotropy on the relaxation modulus of cortical bone would be presented, where relaxation parameters in eq. 18 will be analyzed in conjunction with the structural information of bone.

The bone samples used in this study were obtained from the mid-diaphysis of a 36-monthold bovine femur. Optical microscopic examination showed that all of the samples were generally plexiform but partly transformed into Haversian bone. The samples were cut using a band saw under tap water. In order to examine Young's moduli parallel and normal to the BA, we cut out specimen plates whose longer axes were parallel and normal to the $\mathrm{BA}$, respectively. The cut sections were shaped into rectangular plates with approximate dimension of $0.5 \mathrm{~cm}$ (width), $3.2 \mathrm{~cm}$ (length), and $0.1 \mathrm{~cm}$ (thickness) by using emery paper under tap water. A plate with the longer edge parallel to the BA was coded specimen $\mathrm{P}$, and a plate with the longer edge normal to the BA was coded specimen N. Fig. 17 shows the geometry of specimens.

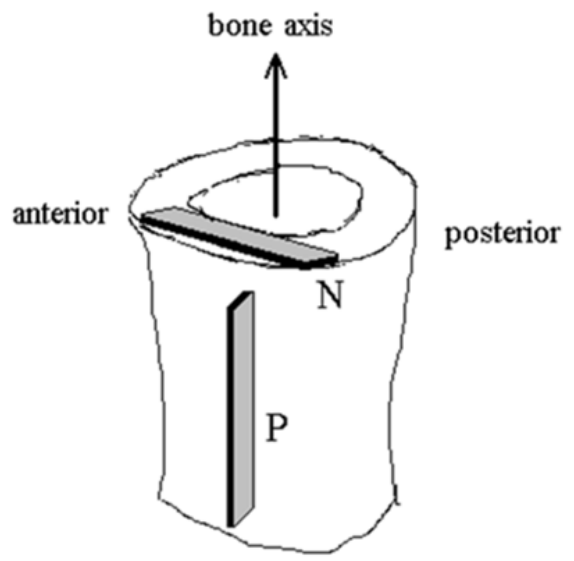

Figure 17. Bone specimens cut parallel $(\mathrm{P})$ and perpendicular $(\mathrm{N})$ to the bone axis.(Iyo et al., J. Biomechanics 37, 1433-1437 (2004). With permission.)

Fig. 18 shows the average values of relaxation Young's modulus, $E(t)$, plotted against time for specimens of $\mathrm{P}$ and $\mathrm{N}$. At several points, standard errors are shown by vertical bars and are listed in Table 2. As mentioned above, stress relaxation of cortical bone has been 
revealed to be expressed by a combination of two relaxation processes according to eq. 18: a fast process (KWW1 process) with a relaxation time, $\tau$, no more than $100 \mathrm{sec}$ and a slow process (KWW2 process) with a relaxation time, $\tau_{2}$, in the order of $10^{6} \mathrm{sec}$. In this experiment, fitting of the average data to eq. 18 was performed. The relaxation modulus results obtained were described well by eq. 18 . The relaxation parameters determined by the fitting, as well as the coefficient of determination, $R^{2}$, and the mean square error, $s$, are listed in Table 2. The average initial value of the relaxation Young's modulus, $E(0)$, of $\mathrm{P}$ was significantly larger than that of $\mathrm{N}(\mathrm{p}<0.05$, ANOVA).
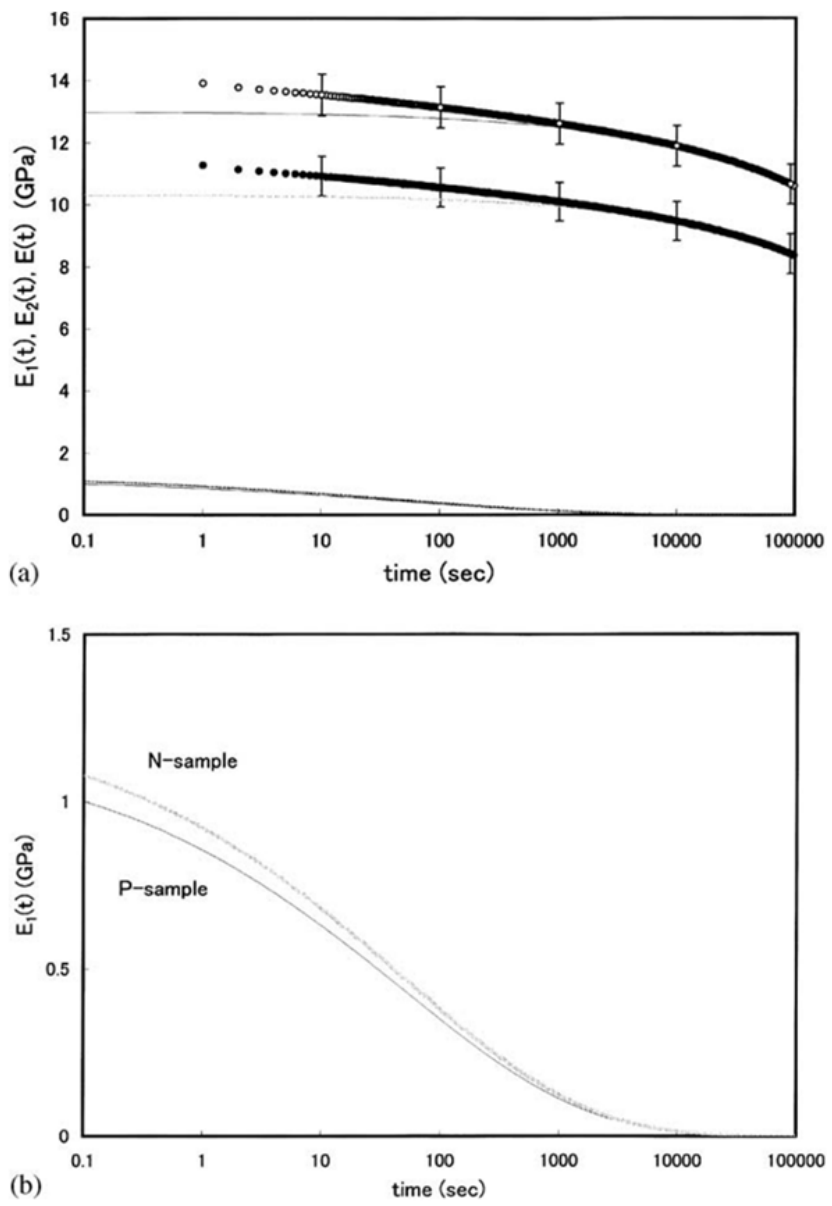

Figure 18. Relaxation modulus curves for $\mathrm{P}(\mathrm{\circ})$ and $\mathrm{N}(\bullet)$ bone specimens. (a) Decomposition into the fast relaxation and slow relaxation processes are shown in lines for each relaxation curve. (b) Magnification of decomposed fast relaxation modulus curves. (Iyo et al., J. Biomechanics 37, 1433-1437 (2004). With permission.) 
In the figure, lines represent the relaxation modulus of the KWW1 process, $E_{1}(t)$, and that of the KWW2 process, $E_{2}(t)$, for $\mathrm{P}$ and $\mathrm{N}$ specimens decomposed from the data according to eq. 18 using parameters of the best fit results listed in Table 2. Despite a difference in structural anisotropy of the specimens, the KWW1 relaxation process of a $\mathrm{P}$ specimen is indistinguishable from that of an $\mathrm{N}$ specimen at this magnification. In order to quantify the anisotropic mechanical properties of cortical bone, anisotropy ratio (AR) has been defined as the ratio of Young's modulus of bone in the direction parallel to the BA, EP, against that normal to the $\mathrm{BA}, E_{\mathrm{N}}, \mathrm{AR}=E_{\mathrm{P}} / E_{\mathrm{N}}$ (Hasegawa et al., 1994). $\mathrm{AR}$ values estimated from our results using average values are listed in Table 3, where the AR value for $E_{0}$ was listed as $\mathrm{AR}_{0}$, and $\mathrm{AR}$ values for $E_{1}, \mathrm{AR}_{1}=E_{\mathrm{P} 1} / E_{\mathrm{N} 1}$, and $E_{2}, \mathrm{AR}_{2}=E_{\mathrm{P} 2} / E_{\mathrm{N} 2}$, were also estimated.

\begin{tabular}{|c|c|c|c|c|c|c|c|c|c|c|c|c|c|}
\hline \multirow[b]{2}{*}{$\begin{array}{c}\text { sample } \\
\text { code }\end{array}$} & \multirow[b]{2}{*}{$\begin{array}{c}\text { sample } \\
\text { size }\end{array}$} & \multirow[b]{2}{*}{$\begin{array}{c}\mathrm{E}_{0} \\
(\mathrm{GPa})\end{array}$} & \multirow[b]{2}{*}{$\mathrm{A}_{1}$} & \multirow[b]{2}{*}{$\tau_{1}(\mathrm{sec})$} & \multirow[b]{2}{*}{$\beta$} & \multirow[b]{2}{*}{$\begin{array}{c}\tau_{2} \\
\left(\mathrm{X} 10^{6} \mathrm{sec}\right)\end{array}$} & \multirow[b]{2}{*}{$\gamma$} & \multicolumn{4}{|c|}{ Standard Error (GPa) } & \multirow[b]{2}{*}{$\mathrm{R}^{2}$} & \multirow[b]{2}{*}{$\mathrm{s}$} \\
\hline & & & & & & & & $10 \mathrm{sec}$ & $10^{2} \mathrm{sec}$ & $10^{3} \mathrm{sec}$ & $10^{4} \mathrm{sec}$ & & \\
\hline $\mathrm{P}$ & 7 & 14.2 & 0.08 & 49 & 0.28 & 9.3 & 0.35 & 0.67 & 0.66 & 0.66 & 0.65 & 0.99989 & 0.0086 \\
\hline $\mathrm{N}$ & 5 & 11.6 & 0.11 & 50 & 0.26 & 6.4 & 0.37 & 0.64 & 0.63 & 0.62 & 0.63 & 0.99986 & 0.0195 \\
\hline
\end{tabular}

(Iyo et al., J. Biomechanics 37, 1433-1437 (2004). With permission.)

Table 2. Relaxation parameters according to the empirical equation (18) determined for the average relaxation modulus curve.

\begin{tabular}{ccccccc}
\hline sample code & $\mathrm{E}_{0}(\mathrm{GPa})$ & $\mathrm{E}_{1}(\mathrm{GPa})$ & $\mathrm{E}_{2}(\mathrm{GPa})$ & $\mathrm{AR}_{0}$ & $\mathrm{AR}_{1}$ & $\mathrm{AR}_{2}$ \\
\hline $\mathrm{P}$ & 14.2 & 1.19 & 13.0 & & & \\
$\mathrm{~N}$ & 11.6 & 1.28 & 10.3 & & & 1.22 \\
\hline
\end{tabular}

(Iyo et al., J. Biomechanics 37, 1433-1437 (2004). With permission.)

Table 3. Decomposition of initial Young's modulus value into those of the KWW1 and KWW2 processes and anisotropic parameters.

The $E_{1}$ value for a $\mathrm{P}$ specimen was almost equal to that for an $\mathrm{N}$ specimen ( $>0.6$, ANOVA), and $\mathrm{AR}_{1}(=0.93)$ was close to 1 , indicating that relaxation Young's modulus in the KWW1 process was insensitive to anisotropic morphology of bone. An elementary process of KWW1 relaxation processes was thought to be attributed to a component of bone that was mechanically isotropic.

$\mathrm{AR}_{2}$ (=1.26) for the KWW2 process was similar to that of $\mathrm{AR}_{0}(=1.22)$ for the whole bone, indicating that an elementary process of the KWW2 relaxation process originates from a component causing the anisotropy of the whole bone. The difference between the whole relaxation Young's modulus value of a $\mathrm{P}$ sample from that of an $\mathrm{N}$ sample is represented by the difference in the respective KWW2 relaxation modulus values. The relaxation time for the KWW2 process, $\tau$ 2, for P-specimen was larger than that for $\mathrm{N}$-specimen. Values of $\gamma$ for $\mathrm{P}$ and $\mathrm{N}$ specimens were similar but larger than $\beta$ values. This indicates that the KWW2 process is attributable to a mode that is governed by the structural anisotropy in bone. 


\subsection{Change in the relaxation modulus of cortical bone by the change in the mineral fraction}

It has been regarded that the stiffness of bone is originated from minerals because modulus value of HAp minerals is almost 100 times larger than that of collagen. In the application as artificial bone materials, materials are required to have bio-compatibility, resistance to corrosion, adequate fracture toughness and fatigue strength. As for the bio-mechanicalcompatibility, in order to obtain the matching in modulus, it is possible to fabricate a composite material of stiff materials with pliant matrix. Changing the stiff component, we will be able to have materials with similar modulus as bone. At the same time, with the change in stiff component, reinforcement state of the matrix can be changed. This means the viscoelastic properties of the matrix changes with the stiff component. We aimed to investigate the viscoelastic properties of bone with changing mineral content (Sasaki \& Yoshikawa, 1993). Demineralization of bone specimens was performed in 0.5 M EDTA, pH8.0 at $4^{\circ} \mathrm{C}$. Mineral fraction was determined by weighing EDTA treated bone specimens. Fig. 19 shows the relaxation moduli for bovine femoral cortical bone specimens of five different mineral contents. The set of relaxation modulus curves appear to be different parts

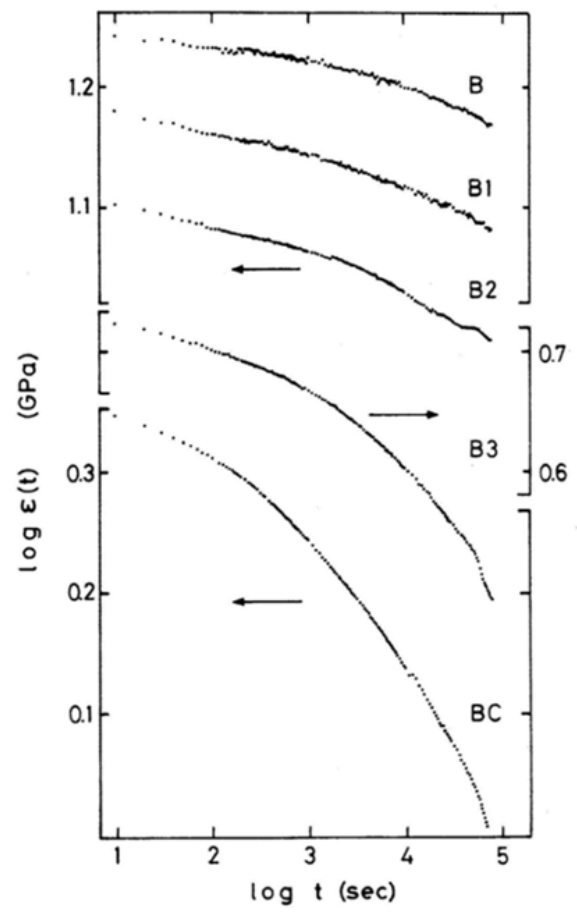

Figure 19. Relaxation modulus curves for bone specimens with various mineral contents; $B \phi_{\mathrm{M}}=0.41$ (volume fraction), B1 $\phi_{\mathrm{M}}=0.35, \mathrm{~B} 2 \phi_{\mathrm{M}}=0.33, \mathrm{~B} 3 \phi_{\mathrm{M}}=0.24$, and BC $=0$ (bone collagen). (Sasaki et al., J. Biomechanics 26, 77-83 (1993). With permission.) 
of a single large master curve, suggesting the reduced variable method, that is, the timemineral content superposition principle could be applicable. Fig. 20 shows the synthetic master curve constructed for bone specimens with different mineral contents. The synthetic curve looks smooth and the scatter of the data points is small. Fig. 21 shows the vertical shift factor, $b_{\mathrm{m}}$, plotted against the mineral content. The filled circles are taken from the mineral content dependence of the elastic modulus of bone after Katz (1971). Mineral content dependence of $b_{\mathrm{m}}$ accords well with that of elastic modulus itself. This fact indicates that the superposition procedure was carried out correctly. Then, the result indicates the timemineral content superposition principle. A polymer-filler system has been considered to have the same reinforcing mechanism as the model discussed. But in the usual polymerfiller system, the time-filler-fraction superposition principle does not hold. The size of the commercially available filler is at least of the order of a few $\mu \mathrm{m}$. By the analysis of the horizontal shift factor, the reinforcing effect depends on the filler-matrix surface area, not on the filler size. The mineral particle in bone, where the time-mineral content reduction was concluded to be applicable, has been recognized to be of the size of a few hundred $\AA$ at most. The reason why the time-filler fraction superposition principle does not hold in the polymer-filler system is deduced to be related to the very large filler size compared with the mineral particles in bone, as well as an adhesive weakness between filler and matrix. This fact leads to the suggestion that, in order to improve the relaxation properties of mineralresin composite as artificial bone, the mineral size should be reduced to, say, submicron level.

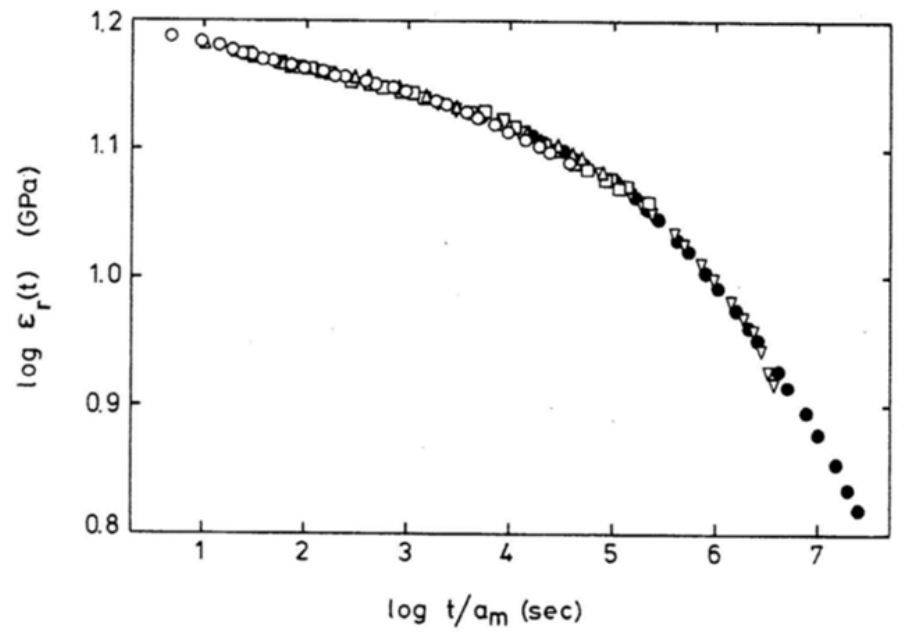

Figure 20. A master curve constructed by superimposing the relaxation modulus curves in Fig. 19. For the successful superposition, both the vertical and horizontal shifts were needed. (Sasaki et al., J. Biomechanics 26, 77-83 (1993). With permission.) 


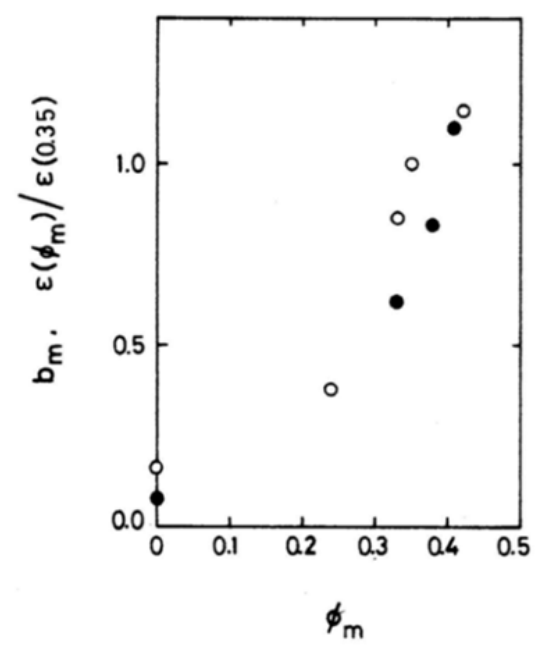

Figure 21. Vertical shift factor as a function of mineral content $\phi_{\mathrm{M}}$ (volume fraction) (०). Mineral content dependence of elastic modulus is also plotted $(\bullet)$. (Sasaki et al., J. Biomechanics 26, 77-83 (1993). With permission.)

\section{Author details}

Naoki Sasaki

Faculty of Advanced Life Science,

Department of Interdisciplinary Sciences, Hokkaido University, Japan

\section{References}

Buschmann, M. D., Gluzband, Y. A., Grodzinsky, A. J., Hunziker, E. B. (1995). Mechanical compression modulates matrix biosynthesis in chondrocyte/agarose culture. Journal of Cell Science 108, 1497-1508.

Currey, JD. (1965) Anelasticity in bone and echinoderm skeletons. Journal of Experimental Biology 43, 279-292.

Currey, J. D. (1964) Three analogies to explain the mechanical properties of bone. Biorheology 2, 1-10.

Fanter, G., Hassenkam, T., Kindt, J., Weaver, J. C., Birkedal, H., Pechenik, L., Cutroni, J. A., Cidade, G. A. G., Stucky, G. D., Morse, D. E., Hansma, P. K. (2005) Sacrificial bonds and hidden length dissipate energy as mineralized fibrils separate during bone fracture. Nature Materials 4, 612-615.

Ferry, J. D. (1980) Viscoelastic Properties of Polymers. Wiley \& Sons, New York.

Foord, S. A., Atkins, E. D. T. (1989). New X-ray diffraction results from agarose: extended single helix structure and implications for gelation mechanism. Biopolymers 28(8), 1345-1365.

Hanazaki, Y., Ito, D., Furusawa, K., Fukui, A., Sasaki, N. (2012) Change in the viscoelastic properties of agarose gel by Hap precipitation by osteoblasts cultured in agarose gel matrix. Journal of Biorhelogy, in press, DOI 10.1007/s12573-011-0043-2. 
Hasegawa, K., Turner, C. H., Burr, D. B. (1994) Contribution of collagen and mineral to the elastic anisotropy of bone. Calcified Tissue International 55, 381-386.

Hayes, W. C., Keer, L. M., Herrmann, G., Mockros, L. F. (1972). A mathematical analysis for indentationtests of articular cartilage. Journal of Biomechanics 5(5), 541-551.

Iyo, T., Sasaki, N., Maki, Y., Nakata, M. (2006) Mathematical description of stress relaxation of bovine femoral cortical bone. Biorheology 43, 117-132.

Iyo, T., Maki, Y., Sasaki, N., Nakata, M. (2004). Anisotropic viscoelastic properties of cortical bone. Journal of Biomechanics 37, 1433-1437.

Jones, W. R., Ting-Beall, H. P., Lee, G. M., Kelly, S. S., Hochmuth, R. M., Guilak, F. (1999). Alterations in the Young's modulus and volumetric properties of chondrocytes isolated from normal and osteoarthritic human cartilage. Journal of Biomechanics 32, 119-127.

Jonscher, A. K. (1983) Dielectric Relaxation in Solids. Chelsea Dielectrics, London.

Kakudo, M., Kasai, N. (1972) X-ray diffraction by polymers. Kodansha, Tokyo.

Katz, J. L. (1971). Hard tissue as a composite material-1. Bounds on the elastic behavior. Journal of Biomechanics 4, 455-473.

Klafter, J., Schlesinger, M. F. (1986) On the relationship among three theories of relaxation in disordered systems. Proceedings of National Academy of Science U. S. A. 83, 848-851.

Lakes, R. S., Katz, J. L., Sternstein, S. S. (1979) Viscoelastic properties of wet cortical bone-I. Tortional and biaxial studies. Journal of Biomechanics 12, 657-678.

Leipzig, N. D., Athanasiou, K. A. (2005). Unconfined creep compression of chondrocytes. Journal of Biomechanics 38, 77-85.

Morita, T., Narita, T., Tokita, M. (2008). Spinodal decomposition of agarose gel. Foods $\mathcal{E}$ Food Ingredients Journal Japan. 213(5), 452-460.

Okazaki, M., Taira, M., Takahashi, J. (1997). Rietveld analysis and Fourier maps of hydroxyapatite. Biomaterials 18(11), 795-799.

Phillips, J. C. (1996). Stretched exponential relaxation in molecular and electric glasses. Reports on Progress in Physics. 59, 1133-1207.

Potuzak, M., Welch, R. C., Mauro, J. C. (2011) Topological origin of stretched exponential relaxation in glass. Journal of Chemical Physics 135, 214502-1-7.

Sasaki, N., Imai, T., Hshimoto, A., Yasuda, H. (2009). Effect of pericellular matrix formation by chondrocytes cultured in agarose gel on the viscoelastic properties of agarose gel matrix. Journal of Biorhelogy, 23, 95-101.

Sasaki, N. (2000) Viscoelastic properties of bone and testing method. In Mechanical testing of bone and the bone-implant interface. (Edited by An, Y. H. and Draughn, R. A.) Boca Raton FL: CRC Press; p. 329-348.

Sasaki, N., Yoshikawa, M. (1993) Stress relaxation in native and EDTA-treated bone as a function of mineral content. Journal of Biomechanics 26, 77-83.

Shieh, A. C., Athanasiou, K. A. (2006). Biomechanics of single zonal chondrocytes. Journal of Biomechanics 39, 1595-1602.

Vincent, J. F. V. (1982) Structural Biomaterials. Macmillan Press, London.

Watase, M., Nishinari, K. (1983) Rheological properties of agarose gels with different molecular weights. Rheologica Acta 22(6), 580-587.

Watase, M., Nishinari, K. (1980) Rheological properties of agarose-gelatin gels. Rheologica Acta 19, 220-225. 